\title{
The Schmidt Law Decoded and Explained
}

\author{
Barry F. Madore \\ The Observatories, \\ Carnegie Institution for Science, \\ 813 Santa Barbara Street, Pasadena CA 91101 \\ email: barry@ociw.org
}

Like the Bode-Titius Law before it, the Schmidt Law is not properly understood, given that it has never been reduced to, or predicted from first principles. Star formation is complicated. And, it is almost certainly a multi-scale process that has multiple immediate causes (quiescent, periodic and impulsive in their time dependence; local, global and cosmic/external in their origin) resulting in the same end product: stars. Philosophers of science would say that the theory of star formation is "massively underdetermined by the observations" of stars alone.

Schmidt (1959) plausibly suggested that the star formation rate (SFR) might scale as some power $n$ of the gas density $\rho$. From dimensional arguments alone Madore (1977) first showed that an exponent of $n=1.5$ would be predicted for gravitationally dominated systems. Sanduleak (1969) empirically tested Schmidt's hypothesis, for the first time in an extragalactic setting, spatially correlating OB stars and neutral hydrogen surface gas densties over the face of the SMC, and determined the power-law exponent to be $n=1.84 \pm 0.14$. Einasto (1972) was so impressed by this determination that he declared Schmidt's hypothesis to be a law, The Schmidt Law. And the rest, as they say, is history.

We have returned to the SMC using larger (photometrically selected) samples of OB stars (Zaritsky 2002) and modern HI maps, but this time using a "process astrophysics" approach to the data. Two astrophysically distinct and observationally unambiguous phases in the star formation process are identified: (1) Regions of a given gas density where (recently-formed. hot, blue) OB stars are currently visible, and (2) those regions, of the same gas density, where no such stars have yet formed or have recently evolved to the red, away from the main sequence and died. The ratio of areas in these two states is directly proportional to the ratio of two independent timescales, $\tau_{*}$ and $\tau_{c c}$. The former (a stellar, astrophysical) timescale is extremely well studied, calibrated and understood; the latter (a hydrodynamic/thermodynamic) timescale is controversial at best.

Expanding upon the mathematical formalism introduced in Madore (2010) where $S F R=M_{*} / \tau=\left(\epsilon M_{g}\right) /\left[\tau_{*}+\tau_{c c}\right]=\left(\epsilon_{o} \rho_{g}^{-\alpha}\right) \times\left(\right.$ Vol. $\left.\times \rho_{g}\right) /\left[\tau_{*}+B \rho_{g}{ }^{-\beta}\right]$ and then $S F R=\left(A \rho_{g}{ }^{1-\alpha}\right) /\left[\tau_{*}+B \rho_{g}{ }^{-\beta}\right]$, we have determined for the SMC that the efficiency of star formation $\epsilon$ varies as $\rho^{-1 / 2}$ and the cloud-collapse timescale $\tau_{c c}$ scales as $\rho^{-5 / 2}$.

\section{References}

Einasto, J. 1972, Ap. Lett., 11, 195

Madore, B. F. 1977, MNRAS, 178, 1

Madore, B. F. 2010, ApJ, 716, L131

Sanduleak, N. 1969, AJ, 74, 47

Schmidt, M. 1959, ApJ, 129, 243

Zaritsky, D., Harris, J., Grebel, E. K., \& Massey, P. 2002, ApJ, 123, 855 